\title{
TECHNO-ECONOMIC COMPARISON OF RENEWABLE ENERGY SYSTEMS USING MULTI-POLE SYSTEM ANALYSIS (MPSA)
}

\author{
MARIO HOLL ${ }^{1}$, MAX F. PLATZER ${ }^{2}$ \& PETER F. PELZ ${ }^{1}$ \\ ${ }^{1}$ Technische Universität Darmstadt, Chair of Fluid Systems, Darmstadt, Germany. \\ ${ }^{2}$ AeroHydro Research \& Technology Associates, Pebble Beach, California, USA.
}

ABSTRACT

The recently published method of multi-pole system analysis (MPSA) is used to techno-economically compare two wind-energy converters: offshore wind turbines and the energy ship concept. According to the method, both systems are (i) modeled, (ii) energetically and economically analyzed, (iii) technoeconomically optimized and, finally, (iv) expected uncertainties are calculated and assessed. The results of the method are used to derive the necessary cost reduction of the wind-energy converters to be economically competitive to fossil-fuel-based technologies.

Keywords: energy ship, holistic system analysis, multi-pole system analysis, techno-economic analysis, technology comparison, wind-energy converter.

\section{INTRODUCTION}

The major share of global renewable energy can be found in ocean regions. Current technological approaches for energy harvesting in these areas concentrate mainly on offshore wind turbines. Nevertheless, offshore wind turbines are currently limited by water depth for reasons of stability, construction, maintenance and accessibility. If one wants to take advantage of ocean wind speeds 'farshore', this thought leads inevitably to mobile wind energy converters. Such a converter has been presented and analyzed by the authors in the past [1-3] and is here referred to as the energy ship concept. A method for holistic system description has been introduced recently by the first and third author [4]. The method was used to simultaneously assess the energetic and economic quality of the concept. In this paper, this method is used to compare offshore turbines and the energy ship concept energetically and economically. Figure 1 shows the compared energy systems with their respective stream tubes. Finally, we answer the question raised by the Global Apollo Programme [5] and present how costs need to reduce to make these renewable energy technologies economically competitive with fossil-fuel-based energy technologies.

\section{MULTI-POLE SYSTEM ANALYSIS (MPSA) OF THE WIND-ENERGY CONVERTERS}

The method of MPSA is a general approach for system evaluation considering arbitrary criteria, e.g. energetic-, mass-, monetary-, or environmental fluxes. The method has been first presented in [4] and consists of the four steps of system (i) modeling, (ii) analysis, (iii) optimization and (iv) sensitivity analysis. The first step shows the interaction of the considered fluxes and leads to a compact and concrete mathematical expression of the considered system. In the second step, the system is analyzed in detail considering the respective fluxes. In step three, the optimum system is derived by stating an objective function. The causal correlations found in step (ii) form the constraints of the optimiza- 

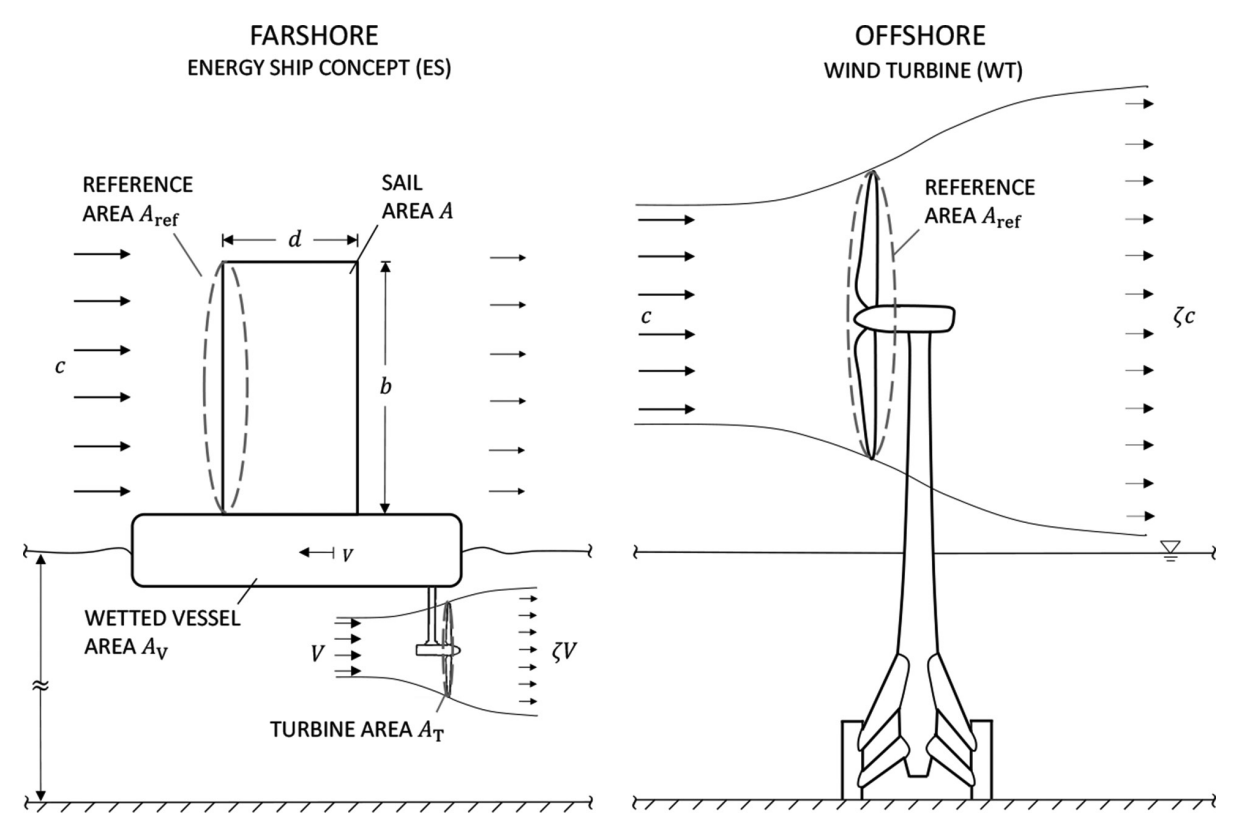

Figure 1: Physical model of the energy ship concept (left) and offshore wind turbine (right).

tion. Thus, one is guided systematically toward a complete optimization problem. The robustness of the optimal solution found is assessed in the last step by performing a sensitivity analysis. In this manner, the uncertainty as well as dominant input factors of the proposed model are identified. These four steps will be gradually applied to the energy ship concept and the offshore wind turbine, to determine their techno-economic quality.

\subsection{System modeling}

A system consists of components, which interact through various fluxes. In the first step, the system is modeled by considering all system components and their respective fluxes using the multi-pole formalism. The fluxes are listed in a column matrix, whereas the components are specified by square matrices. Series connected components are combined by multiplying all matrices, whereas parallel connected components are combined by the summation of the respective matrices. Thus, every arbitrary interconnected system can be presented in the notation $\underline{x}=\Omega \underline{y}$, with the input fluxes $\underline{x}$, the output fluxes $\underline{y}$ and the system matrix $\Omega$. The multi-pole model as well as the mathematical representation of the wind turbine and energy ship are shown in Table 1. It can be seen that the multi-pole model of the energy ship also contains mass fluxes. This is necessary, since the mobile energy converter stores the converted electricity chemically through the electrolytic splitting of water into hydrogen.

A detailed consideration of the multi-pole model of the energy ship can be found in [4] and will not be further addressed here. As one can see from the multi-pole models, all components are series connected and, thus, all matrices can be multiplied so that one ends up with the respective compact system representation 
Table 1: Multi-pole model of the energy ship concept and offshore wind turbine.

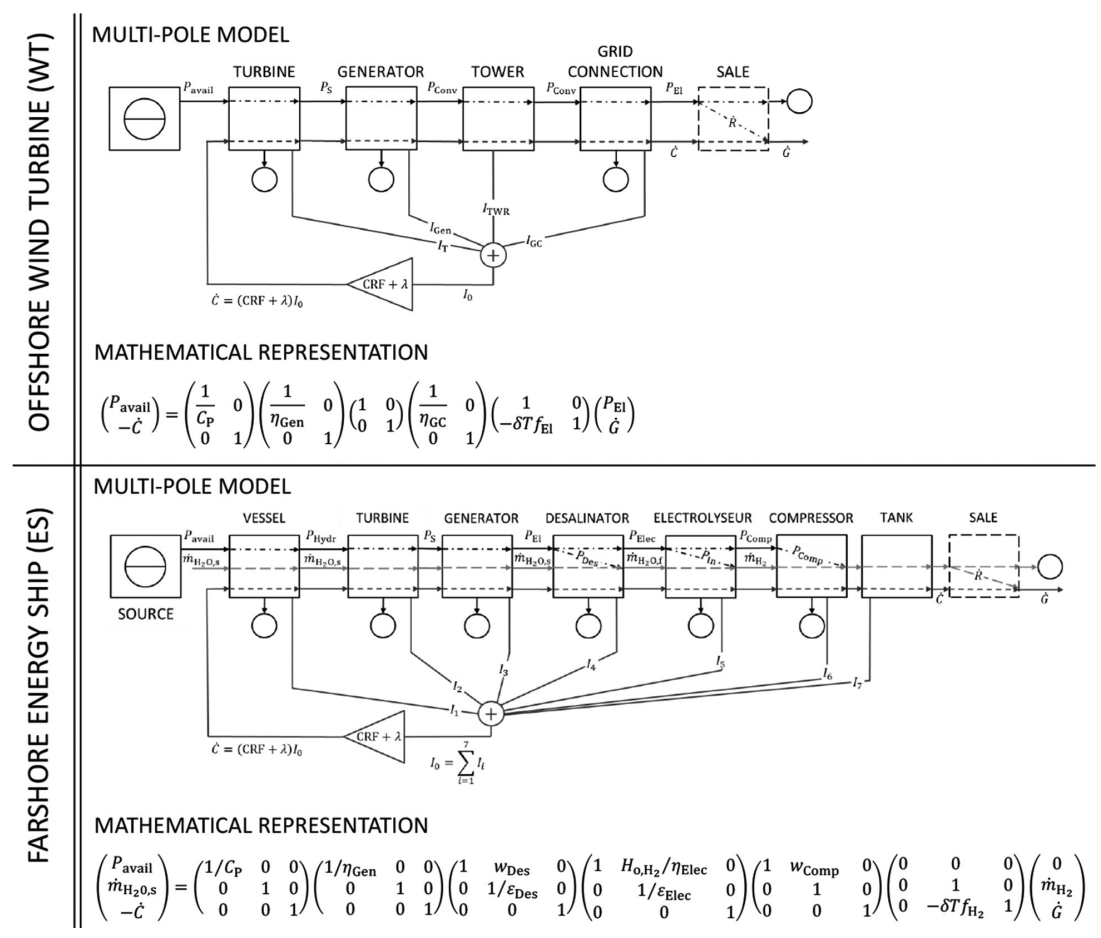

$$
\begin{aligned}
& \left(\begin{array}{l}
P_{\mathrm{avai}} \\
-\dot{C}
\end{array}\right)_{\mathrm{WT}}=\left(\begin{array}{cc}
\frac{1}{C_{\mathrm{P}} \eta_{\mathrm{Gen}} \eta_{\mathrm{GC}}} & 0 \\
-\delta T f_{\mathrm{El}} & 1
\end{array}\right)_{\mathrm{WT}}\left(\begin{array}{c}
P_{\mathrm{El}} \\
\dot{G}
\end{array}\right)_{\mathrm{WT}},
\end{aligned}
$$

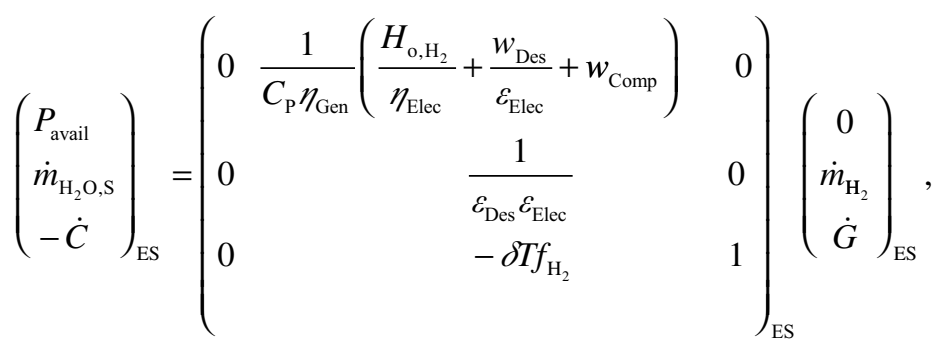

with the abbreviations WT for wind turbine and ES for energy ship. Power is denoted as $P$, efficiency factors as $\eta$, mass conversion rates as $\varepsilon$, mass-specific work as $w$, mass flow as $\dot{m}$, periodic costs as $\dot{C}$ and periodic profit as $\dot{G}$. The capacity factor $\delta$ is defined as the yearly energy output in relation to the energy output produced at full capacity. It can be seen that the energy ship provides hydrogen, whereas the wind turbine provides electric power. The hydrogen and electricity market price is denoted as $f_{\mathrm{El}}$ or $f_{\mathrm{H}_{2}}$, respectively. The coupling mechanism is illustrated for both systems by the fact that the more revenue is gained the more efficiently the respective system operates. This can be seen directly in the above equations. 


\subsection{Detailed system analysis}

Since both systems are compared techno-economically, meaning by the simultaneous consideration of energetic and economic aspects, the detailed system analysis can be separated into an energetic and economic analysis.

\subsubsection{Energetic analysis}

The energetic analysis is limited here to the consideration of the respective fluid systems. A fluid system consists always of an in- and outflow and the machinery in between, as shown in Fig. 1. In 1920, Betz [6] defined a quantity called Coefficient of Performance $C_{\mathrm{P}}$ for wind turbines. Therefore, he assumed a hypothetical machine, which extracts the total available power $P_{\text {avail,wT }}:=\varrho_{\mathrm{g}} c^{3} A_{\text {ref }} / 2$ where the air density is $\varrho_{\mathrm{g}}$, the absolute wind speed is $c$ and the circular rotor area is $A_{\text {ref }}$ (see Fig. 1). Treating the turbine as an ideal disc actuator, he derived the mechanical turbine shaft power using the condition of mass-, momentum- and energy conservation $P_{\mathrm{S}, \mathrm{WT}}=\varrho_{\mathrm{g}} c^{3} A_{\mathrm{ref}} \eta_{\mathrm{T}}\left(1-\zeta^{2}\right)(1+\zeta) / 4$. The ratio of the available power and the mechanical turbine power is defined as the coefficient of performance

$$
C_{\mathrm{P}, \mathrm{WT}}:=\frac{P_{\mathrm{S}, \mathrm{WT}}}{P_{\text {avail } \mathrm{WT}}}=\frac{1+\zeta}{2}\left(1-\zeta^{2}\right) \eta_{\mathrm{T}},
$$

with $\zeta$ as the ratio of the far upstream and downstream velocities. For $\zeta=1 / 3$ and an ideal turbine $\left(\eta_{\mathrm{T}}=1\right)$ the coefficient of performance maximizes and becomes the famous Betz' law $C_{\text {P,WT,opt }}=16 / 27$, an axiomatic based physical upper limit for energy conversion of wind turbines. The fluid system of the energy ship is analyzed in the same way. Therefore, we define the available power as $P_{\text {avail,Es }}:=\varrho_{\mathrm{g}} c^{3} A_{\text {ref }} / 2$, where $A_{\text {ref }}$ is the circular area with the diameter $b$, which is the span of the sail. This area is used because the sail physically influences the air mass going through this area. As Prandtl showed, the lift of a wing is equal to this air mass multiplied with the induced downwash velocity [7]. In analogy to Betz, the hypothetical machine is thus the one, which extracts all power going through this area. The mechanical turbine shaft power is also calculated using mass-, momentum- and energy conservation $P_{\mathrm{S}, \mathrm{ES}}=\varrho_{1}(V(\zeta))^{3} A_{\mathrm{T}} \eta_{\mathrm{T}}\left(1-\zeta^{2}\right)(1+\zeta) / 4$. Note the change of the medium since $\varrho_{1}$ denotes the water density and $V$ is the vessel speed, which is a function of the ratio of the far upstream and downstream velocities $\zeta$. The coefficient of performance can be calculated as

$$
C_{\mathrm{P}, \mathrm{ES}}:=\frac{P_{\mathrm{S}, \mathrm{ES}}}{P_{\mathrm{avail}, \mathrm{ES}}}=\frac{4}{\varrho \pi \Lambda}(v(\zeta))^{3} a_{\mathrm{T}} \frac{1+\zeta}{2}\left(1-\zeta^{2}\right) \eta_{\mathrm{T}},
$$

with the dimensionless vessel speed $v:=V / c$, the dimensionless density ratio $\varrho:=\varrho_{\mathrm{g}} \varrho_{1}$ the dimensionless turbine area $a_{\mathrm{T}}:=A_{\mathrm{T}} / A$ and the aspect ratio $\Lambda:=b / d$ according to Fig. 1. It is physically reasonable that $C_{\mathrm{P}, \mathrm{ES}}<C_{\mathrm{P}, \mathrm{WT}}$, since a part of the available wind power is used for vessel propulsion.

\subsubsection{Economic analysis}

For the economic analysis, the common method of net present value NPV is used with the periodic monetary flow $\dot{Z}_{t}$ and the rate of interest $z$. If the initial investment is excluded from the sum shown in eqn. (5) and the remaining monetary flows are considered as periodic constant revenues $\dot{R}$ and periodic constant costs, calculated as a percentage of the initial investment $\lambda I_{0}$, the NPV reads 
Table 2: Cost and revenue analysis of the wind turbine and the energy ship concept.

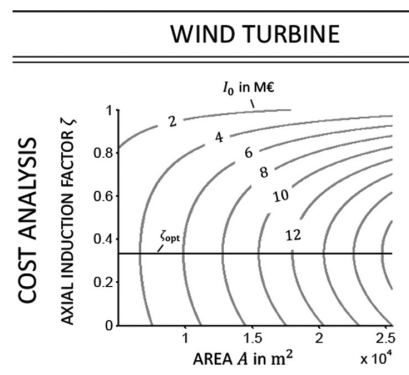
ENERGY SHIP

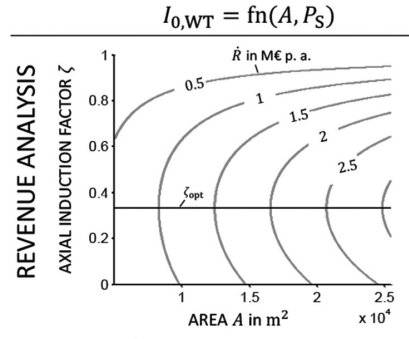

$\dot{R}_{\mathrm{WT}}=\delta_{\mathrm{WT}} T P_{\mathrm{El}} f_{\mathrm{El}}$
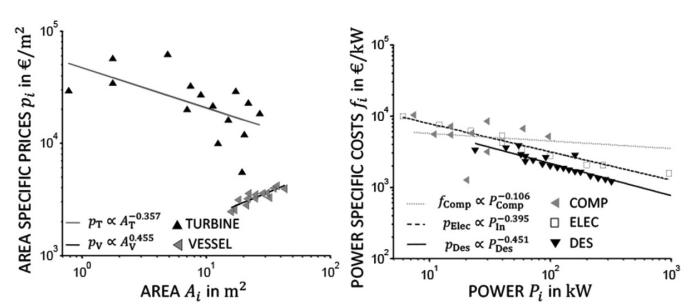

$I_{0, \mathrm{ES}}=\mathrm{fn}\left(A_{i}, P_{i}\right)$

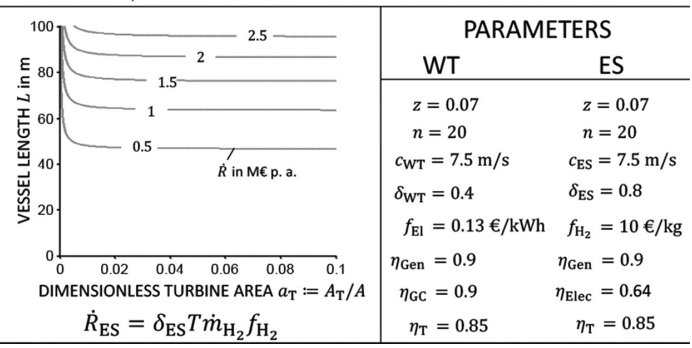

$$
\mathrm{NPV}=\sum_{t=0}^{n} \frac{\dot{\mathrm{Z}}_{t}}{(1+z)^{t}}=-I_{0}+\sum_{t=1}^{n} \frac{\dot{R}-\lambda I_{0}}{(1+z)^{t}}=-I_{0}+\frac{1}{\mathrm{CRF}}\left(\dot{R}-\lambda I_{0}\right)
$$

with the capital recovery factor $\mathrm{CRF}=z(1+z)^{n} /\left((1+z)^{n}-1\right)$ and the lifetime $n$. This equation can be written as the periodic profit function

$$
\underbrace{\mathrm{CRF} \cdot \mathrm{NPV}}_{\dot{G}}=\dot{R}-\underbrace{(\mathrm{CRF}+\lambda) I_{0}}_{\dot{C}} .
$$

Table 2 shows the cost scaling functions of the offshore wind turbine and the energy ship concept. The scaling functions of the energy ship have been derived on the basis of literature and market surveys. The scaling functions of the offshore wind turbine are based on the work of Engels [8]. Engels also uses empirical scaling functions to describe the costs as a function of the rotor area and the rated power.

One can see that the costs of the wind turbine become a maximum for the optimal operation, since the rated power becomes a maximum. Also, the yearly revenue maximizes for the optimal turbine operation and maximal turbine rotor area, because more electricity can be sold. The detailed cost scaling functions are explained in more detail in $[4,8]$, respectively. The most revenue is gained for an energy ship with maximal vessel length and maximal turbine area.

\subsection{System optimization}

The next step of the MPSA method deals with the system optimization. Both systems have been modeled, mathematically combined and analyzed under energetic and economic aspects using empirical scaling laws. Thus, a multitude of possible systems is described. Through 
the process of system optimization, the optimal system of this multitude is identified. The energetic and economic models presented here are highly non-linear. Since empirical scaling functions are used, the system can be described by continuous functions. Thus, the optimization problems can be assigned to the class of non-linear continuous optimization functions. The output uncertainty, caused by uncertain input factors and the scaling uncertainty, will be addressed in the fourth step. In this paper, the techno-economic optimal offshore wind turbine and energy ship are derived.

An optimization problem consists always of an objective function subjected to constraints. The constraints describe the specifications of the system, e.g. the physical coherences of the respective system. Consequently, all analytic equations found in the detailed system analysis are part of the optimization constraints. The levelized costs of electricity LCOE and the levelized costs of hydrogen $\mathrm{LCOH}$ are used as objective function. They are defined as the critical product price, which assures periodic cost neutrality. A greater market price than the levelized costs will lead to economic profit, whereas a smaller market price leads to economic losses. Thus, one will pursue the minimization of the levelized costs. The optimization problem as well as the optimal designs, as the results of the optimization, are presented in Table 3.

It can be seen that the smallest LCOE of the wind turbine are gained for optimal system operation $\zeta=1 / 3$ and a specific rotor area of $A_{\mathrm{WT}, \mathrm{opt}}=1.58 \cdot 10^{4} \mathrm{~m}^{2}$, corresponding to a diameter of $D=142 \mathrm{~m}$. The minimal LCOE can be specified to LCOE $=12.46 €$ ct. $/ \mathrm{kWh}$. The optimal dimensionless turbine area $a_{\mathrm{T}, \mathrm{opt}}$ and the optimal vessel length of the energy ship concept can also be seen in Table 3 and correspond to the results presented in [4]. The plot

Table 3: Mathematical optimization problem and graphical representation of the respective optimal system design and operation.

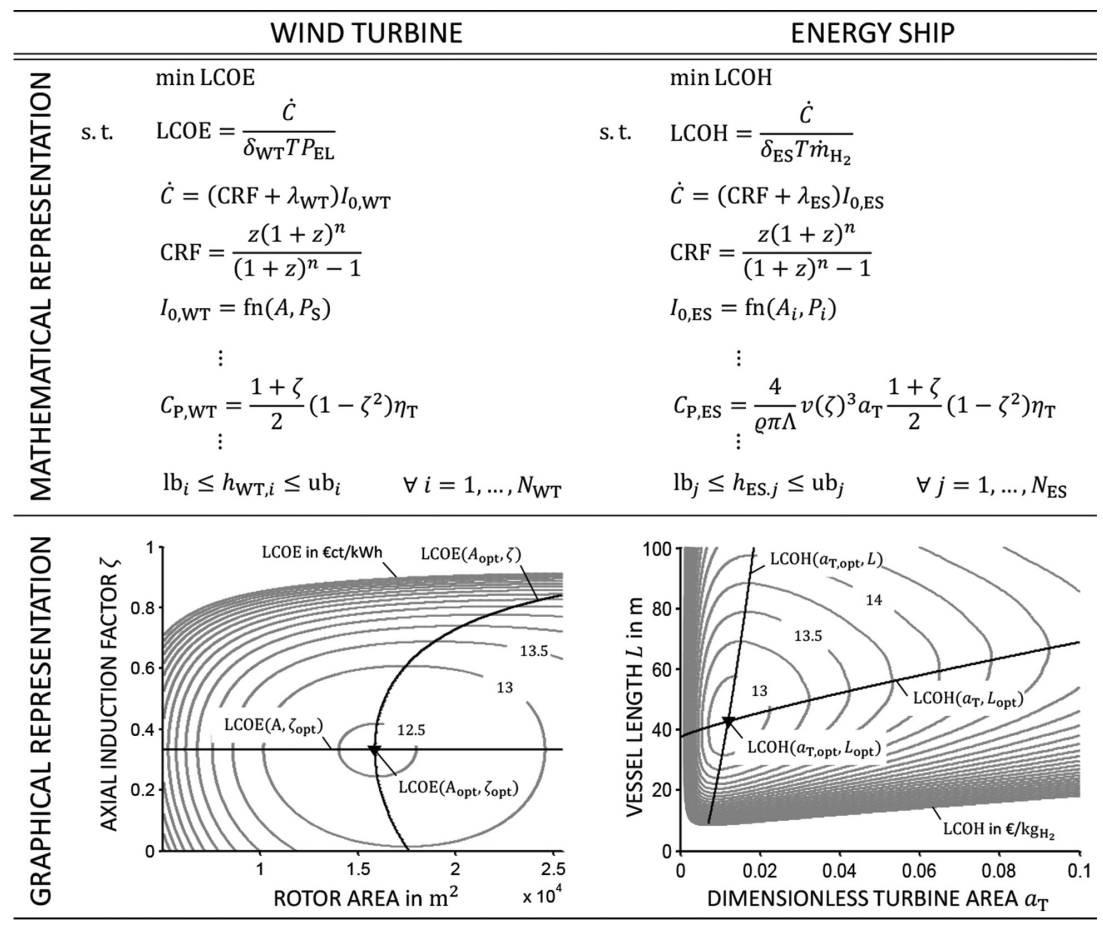


has been created with the optimal operation of the energy converter. The minimal LCOH can be specified to $\mathrm{LCOH}=12.83 € / \mathrm{kg}_{\mathrm{H}_{2}}$. Thus, using economic and energetic aspects simultaneously, the optimal design and operation of the respective energy converter can be calculated.

\subsection{Sensitivity analysis}

A sensitivity analysis is performed to assess the robustness of the previously found optimal system and to ensure the overall quality of the modeling process. Therefore, input factors are no longer considered as deterministic variables, but as random variables with a specific distribution. Through an uncertainty analysis, the output uncertainty is calculated using Monte-Carlo-Simulations. By applying an appropriate method of sensitivity analysis, the output uncertainty is assigned to the different sources of input uncertainty. Thus, all input factors can be listed according to their influence. This process is called factor prioritization. In [9], the authors presented a detailed sensitivity analysis of the energy ship concept using variance-based sensitivity analysis and the recently published PAWN method [10, 11]. The PAWN method will be used here and belongs to the density-based methods of sensitivity analysis, considering the entire output distribution. Through Monte-Carlo-Simulations, the unconditional cumulative distribution function $\operatorname{CDF} F Y(y)$ is calculated with the output random variable $Y$. In the next step, the conditional $\operatorname{CDF} F_{Y \mid X_{i}=x_{i}^{*}}(y)$ is calculated, where the random variable $X_{i}$ is fixed to the realization $x_{i}^{*}$. The so-called Kolmogorov-Smirnov statistic $\mathrm{KS}$ is defined as the maximal distance of unconditional and conditional CDFs for the realization $x_{i}^{*}$

$$
\operatorname{KS}\left(x_{i}^{*}\right)=\max _{y}\left|F_{Y}(y)-F_{Y \mid X_{i}=x_{i}^{*}}(y)\right| .
$$

The sensitivity index $T_{i}$ of the random input variable $X_{i}$ is defined as the median of all $\mathrm{KS}$ statistics for all realizations

$$
T_{i}:=\operatorname{median}_{x_{i}^{*}=x_{i, 1}^{*} \cdots x_{i, N}^{*}}\left(\operatorname{KS}\left(x_{i}^{*}\right)\right) .
$$

The dependence on the realization $x_{i}^{*}$ is lost since the median is used. The sensitivity index $T_{i}$ varies between 0 and 1 , whereas a high value represents a high influence and vice versa. The input uncertainties are modeled with the probability density functions PDF of a normal distribution $\mathcal{N}(\mu, \sigma)$ characterized by its expected value $\mu$ and the standard deviation $\sigma$, the uniform distribution $\mathcal{U}(a, b)$ with the lower value $a$ and upper value $b$ and the $t$ distribution $\mathcal{T}(\phi)$, specified by the number of samples $\phi$. The input factors with their respective PDF, the result of the uncertainty analysis as well as the result of the sensitivity analysis are shown in Table 4.

It can be seen that the optimal levelized costs are obtained with a probability of approximately $50 \%$. The most dominant input factors of the wind turbine model are the capacity factor $\delta$ and the costs, specified by the initial investment $I_{0}$ and the O\&M costs $\lambda I_{0}$. In contrast, the LCOH uncertainty of the energy ship is mainly driven by the uncertainty of the performance parameters lift and drag coefficient $c_{\mathrm{L}}$ and $c_{\mathrm{D}}$. A more detailed sensitivity consideration of the energy ship model can be found in [9]. 
Table 4: Results of the uncertainty and sensitivity analysis and listing of the uncertain input factors.

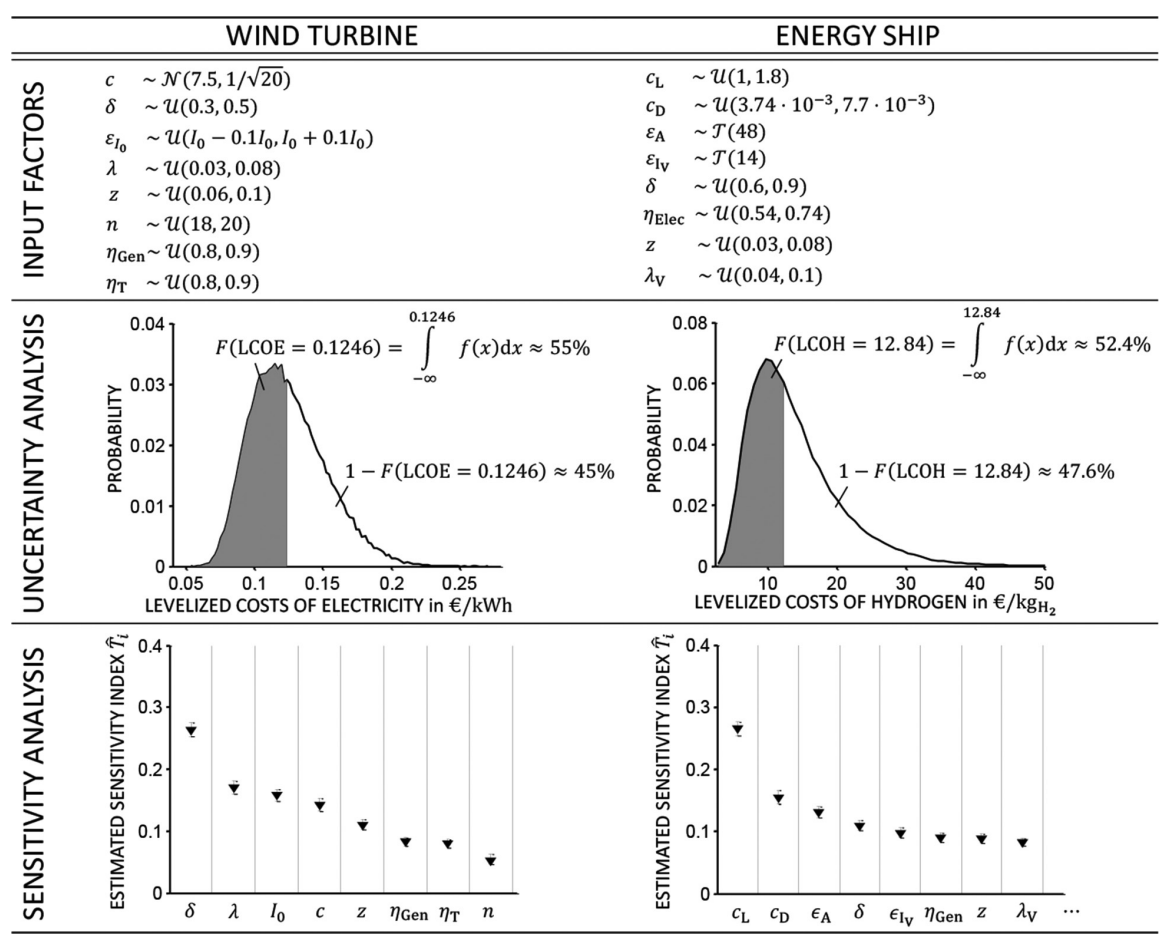

\section{NECESSARY COST REDUCTION FOR ECONOMIC COMPETITIVENESS}

After evaluating the systems techno-economically, the obtained results can be used to answer the question raised by the recently established Global Apollo Programme: "How do investment costs need to reduce to make renewable energy systems economically competitive with fossil fuel based energy technologies?"

A technology is economically competitive if, in general, the levelized costs of the product $X(\mathrm{LCO} X)$ have the same order as the target $\mathrm{LCO}_{\text {tar }}$ of established technologies in the respective markets. Thus, if economic competitiveness is required, one requires mathematically

$$
\mathrm{LOC} X \stackrel{!}{=} \mathrm{LCO} X_{\text {tar }}
$$

If the definition of the LCOX in Table 3 is recalled, this equation reads

$$
\frac{(\mathrm{CRF}+\lambda) I_{0, \operatorname{tar}}}{\delta T X}=\mathrm{LCO} X_{\mathrm{tar}},
$$

with the target investment costs $I_{0, \operatorname{tar}}$, which can be calculated to $I_{0, \operatorname{tar}}=\mathrm{LCO} X_{\operatorname{tar}} \cdot \delta T X /(\mathrm{CRF}+\lambda)$. The difference of the actual investment costs $I_{0 \text {,act }}$ and the target investment costs is the necessary cost reduction.

$$
I_{0, \text { red }}=I_{0, \text { act }}-I_{0, \text { tar }}
$$


Table 5: Actual and target levelized costs and necessary relative cost reduction RCR for wind turbine and the energy ship.
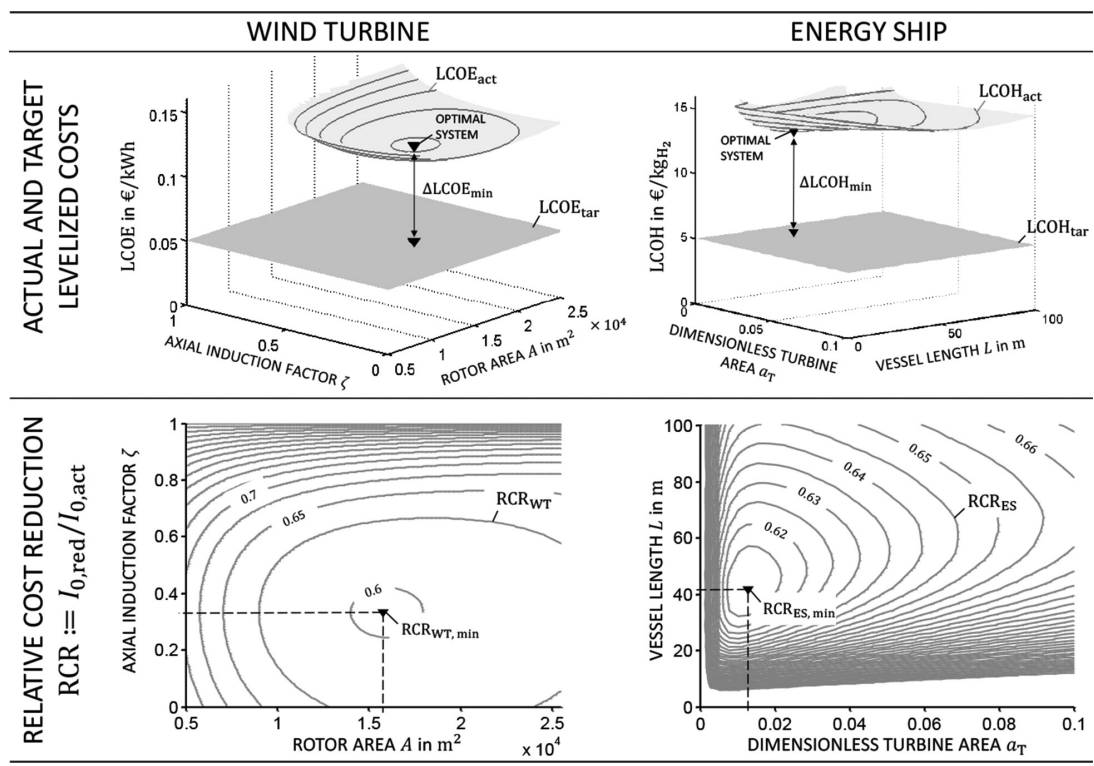

By dividing this equation by the actual investment costs, a dimensionless measure for the necessary cost reduction is obtained. We define this dimensionless measure as the relative cost reduction RCR.

$$
\mathrm{RCR}:=\frac{I_{0, \mathrm{red}}}{I_{0, \mathrm{act}}}=1-\frac{I_{0, \mathrm{tar}}}{I_{0, \mathrm{act}}} .
$$

The cost reduction is expressed as multiples of the actual investment costs. We compare the LCOE of the wind turbine with the LCOE of a coal-fired power plant, specified by $\mathrm{LCOE}_{\text {tar }}=0.05 € / \mathrm{kWh}$ (which is the current LCOE in Germany, 2016). The energy ship concept is compared with fossil-fuel-based hydrogen producing technologies with LCOH varying between $2-6 € / \mathrm{kg}_{\mathrm{H}_{2}}$. In this paper, we assume $\mathrm{LCOH}_{\text {tar }}=5 € / \mathrm{kg}_{\mathrm{H}_{2}}$. Table 5 shows the results. In the first row, the respective target and the actual levelized costs can be seen. One can expect the necessary cost reduction to become a minimum for the respective optimal techno-economic system, since the difference of the actual and target levelized costs $\triangle \mathrm{LCOX}$ become a minimum. In the second row of Table 5, the RCR of wind turbine and energy ship concept can be seen. As expected, the cost reduction becomes a minimum for the techno-economic optimal system design. As the minimal $\mathrm{RCR}_{\mathrm{WT}} \approx 0.6$ indicates, the investment costs for offshore wind turbines must be reduced by $60 \%$ of the actual investment costs in order to be economically competitive with coal-fired power plants. The investment costs of the energy ship must be reduced by $\mathrm{RCR}_{\mathrm{ES}} 61 \%$ of the actual investment costs in order to be economically competitive to a hydrogen providing technology, specified by $\mathrm{LCOH}_{\mathrm{tar}}=5 € / \mathrm{kg}_{\mathrm{H}_{2}}$.

\section{CONCLUSION}

The MPSA method is used for the first time to compare renewable energy systems. The consideration is limited to energetic and economic aspects and, thus, can be referred to as a 
techno-economic comparison. Through the four steps of the MPSA method, a superordinate model presented in step (i) is derived for each system, showing linkages and interactions of energetic and economic aspects. The systems are analyzed energetically and economically in detail in step (ii) of the method using empirical scaling laws. In the (iii) step of the method, the respective techno-economic optimal systems are derived. The robustness, the total output uncertainty and dominant input factors of the optimal systems found are assessed in the (iv) step by applying methods of sensitivity analysis. Through the application of the MPSA method, the systems can be considered as White-Box models concerning the modeled criteria. Thus, any questions raised by the use of these criteria can be answered using the results of the MPSA method. In this paper, we answer the question raised by the Global Apollo Programme by deriving the necessary relative cost reduction for each technology to become economically competitive with established technologies.

\section{ACKNOWLEDGMENTS}

The authors would like to thank the Deutsche Forschungsgemeinschaft (DFG) for the financial support in the framework of the Excellence Initiative, Darmstadt Graduate School of Excellence Energy Science and Engineering (GSC 1070).

\section{REFERENCES}

[1] Holl, M., Platzer, M. \& Pelz, P.F., Optimal energy systems design applied to an innovative ocean-wind energy converter. Proceedings of the 7th International Conference on Sustainable Development and Planning, WIT Press: Istanbul, Turkey, pp. 547-557, 2015. http://dx.doi.org/10.2495/sdp150471

[2] Holl, M., Platzer, M. \& Pelz, P.F., Techno-economical system optimisation and its application to an energy system. Energy-Science and Technologie EST, KIT Karlsruhe: Karlsruhe, Germany, p. 406, 2015.

[3] Pelz, P.F., Holl, M. \& Platzer, M., Analytical method towards an optimal energetic and economical wind-energy converter. Energy, 94, pp. 344-351, 2016. http://dx.doi.org/10.1016/j.energy.2015.10.128

[4] Holl, M. \& Pelz, P.F., Multi-pole system analysis (MPSA) - A systematic method towards techno-economic optimal system design. Applied Energy, 169, pp. 937-949, 2016. http://dx.doi.org/10.1016/j.apenergy.2016.02.076

[5] King, D., Browne, J., Layard, R., O’Donnell, G., Rees, M., Stern, N. \& Turner, A., A global apollo programme to combat climate change. Report, LSE - The London School of Economics and Political Science, 2015.

[6] Betz, A., Das Maximum der theoretisch möglichen Ausnützung des Windes durch Windmotoren. Zeitschrift für das gesamte Turbinenwesen, 26, pp. 307-309, 1920.

[7] Tietjens, O.K.G. \& Prandtl, L., Applied Hydro- and Aeromechanics: Based on Lectures of L. Prandtl, volume 2, Courier Corporation, 1957.

[8] Engel, P., Berechnung der optimalen Auslegung von Offshore-Windkraftanlagen zur Erhöhung der Versorgungssicherheit. Dissertation, Technische Universität Darmstadt, 2014.

[9] Holl, M., Janke, T., Pelz, P.F. \& Platzer, M., Sensitivity analysis of a techno-economic optimal wind-energy converter. Proceedings of the 2nd International Conference on Next Generation Wind Energy, Lund, Sweden, 2016. 
[10] Pianosi, F., Sarrazin, F. \& Wagener, T., A Matlab toolbox for Global Sensitivity Analysis. Environmental Modelling \& Software, 70, pp. 80-85, 2015. http://dx.doi.org/10.1016/j.envsoft.2015.04.009

[11] Pianosi, F. \& Wagener, T., A simple and efficient method for global sensitivity analysis based on cumulative distribution functions. Environmental Modelling \& Software, 67, pp. 1-11, 2015.

http://dx.doi.org/10.1016/j.envsoft.2015.01.004 